\title{
Amphiregulin as a novel diagnostic and prognostic biomarker of hepatocellular carcinoma before and after locoregional treatment
}

Amira Isaac ${ }^{*}$ D, Sherif Mounir Mohamed, Osama Ashraf Ahmed, Ahmed Gamal Mohamed Hassan and Hany Samir Rasmy

\begin{abstract}
Background: Hepatocellular carcinoma is a highly prevalent tumor worldwide. Amphiregulin is a ligand of the epidermal growth factor receptor. Its elevation is linked to different inflammatory and neoplastic conditions. Therefore, amphiregulin may represent a potential diagnostic target in HCC, which has sparked interest as a potential predictor of diagnosis and progression of HCC. The current work was set out to evaluate amphiregulin as a possible diagnostic and prognostic biomarker for HCC on top of cirrhosis. Thirty adult patients with liver cirrhosis and HCC (HCC group) were randomly selected as candidates for locoregional therapies, either radiofrequency ablation or transarterial chemoembolization. A separate group of thirty liver cirrhosis patients served as controls (cirrhosis group). All patients underwent standard laboratory tests and abdominal ultrasounds. Alpha-fetoprotein and amphiregulin were measured twice at baseline and 1 month after the intervention.
\end{abstract}

Results: Baseline serum amphiregulin was significantly higher in the HCC group than in the cirrhosis group $(23.2 \pm 11.5$ vs. $11.1 \pm 7.1)$, with a $p$ value $<0.001$. Patients with multiple and larger focal lesions had greater levels of amphiregulin, with $p$ values of 0.015 and 0.002 , respectively. At 1 month following locoregional treatment, the amphiregulin level considerably declined compared with its baseline levels (from 23.2 \pm 11.5 to 19.4 \pm 10.9 ), with a $p$ value of 0.012 , while AFP showed an insignificant reduction. At follow-up, the level of serum amphiregulin was statistically significantly greater in recurrence cases than in remission cases ( $30.8 \pm 14.1$ vs. $17.2 \pm 8.8$ ), with a $p$ value of 0.008 , and the same was observed for AFP level.

At a cutoff $\geq 17 \mathrm{pg} / \mathrm{mL}$, amphiregulin was a valuable marker in HCC detection with a sensitivity and specificity of $63.3 \%$ and $86.7 \%$, respectively, while it has $60 \%$ sensitivity and $96 \%$ specificity in detecting possible tumor recurrence at a cutoff $\geq 29.7 \mathrm{pg} / \mathrm{ml}$.

Conclusions: Amphiregulin may be a good diagnostic marker for HCC and a prognostic marker after locoregional therapies because its follow-up levels are useful in predicting possible tumor recurrence.

Keywords: Amphiregulin, Hepatocellular carcinoma, Radiofrequency ablation, Transarterial chemoembolization

*Correspondence: dr.amira@med.asu.edu.eg

Department of Internal Medicine, Gastroenterology and Hepatology Unit,

Faculty of Medicine, Ain Shams University, Cairo 11566, Egypt

\section{Background}

Hepatocellular carcinoma (HCC) is rising and represents the third leading cause of malignancy-related deaths [1].

Most HCC cases develop in chronic hepatic inflammation due to viral hepatitis, alcohol intake, and 
non-alcoholic steatohepatitis (NASH). However, the HCC mechanism has not been fully understood [2].

Long-term survival is best achieved through tumor resection and liver transplantation. Nevertheless, surgical resection is not achievable in more than $80 \%$ of cases due to tumor site, size, and underlying disease severity [3].

Locoregional therapy is regarded as the most important line of HCC treatment, including radiofrequency ablation (RF), which is a better treatment alternative for early-stage HCC patients who are ineligible for surgery or transplantation with minimal rates of recurrence and higher rates of disease-free survival [4].

Transarterial chemoembolization (TACE) has been demonstrated to enhance survival in patients with maintained liver function, especially those with ChildPugh class A cirrhosis who do not have vascular invasion, extrahepatic metastases, or severe cancer-related symptoms. TACE can also be utilized as a neoadjuvant treatment or to downstage patients before liver transplantation [5].

The current methods for HCC diagnosis, whether radiological or laboratory biomarkers, are ineffective, especially for detecting small and early lesions [6].

Alpha-fetoprotein (AFP) is the most popular marker for HCC detection, with a $40 \%$ false-negative rate when used alone for early-stage HCC. Its value may still be normal in 15 to $30 \%$ of patients, even those with latestage HCC [3].

The epidermal growth factor receptor (EGFR) pathway is a prominent pathway involved in various cellular functions, and its dysregulation is a common theme in cancer biology [7].

Amphiregulin (AREG) is an EGFR ligand, a transmembrane tyrosine kinase with a wide expression. It is made as a membrane-bound precursor protein. Via EGFR binding, AREG stimulates major intracellular signaling pathways that govern cell proliferation and survival. Chronic AREG expression has been associated with several pathological conditions, and the majority of which are inflammatory and/or neoplastic. Consequently, AREG may represent a potential diagnostic target in $\mathrm{HCC}$, which has received much interest as a potential predictor of the presence and progression of HCC [8].

The present work aims to assess the diagnostic value of AREG for HCC and its prognostic value following various locoregional therapeutic modalities.

\section{Methods}

This study included 30 randomly chosen adults with liver cirrhosis and HCC due to HCV (HCC group). According to 2011 AASLD guidelines, HCC was diagnosed based on the development of characteristic vascular enhancement in triphasic abdominal CT scans [9]. Further, a control group of 30 age- and gender-matched patients (cirrhosis group) with liver cirrhosis without HCC was included in the trial; the HCC was excluded based on the absence of any hepatic focal lesions by triphasic CT. The study involved patients who attended the hepatology outpatient clinics between January and October 2020.

Our selection eliminated patients with contraindications to locoregional treatment modalities, including major portal vein branch invasion, other malignancies, or extrahepatic metastases, Child $C$, platelet count less than $50 \times 10^{9} / \mathrm{L}$, or prothrombin activity less than $50 \%$.

The study was following the ethical principles of the 1975 Helsinki Declaration that was granted by the local Ethics Committee (FWA 000017585). Before being enrolled in the trial, participants gave their informed written consent after being informed about the study's goal and required interventions.

All patients underwent the following: full detailed history, a thorough examination with particular focus on the existence of signs of chronic liver disease, and laboratory tests including complete blood count, full hepatic profile, serum creatinine, HCVAb, and HBsAg. Child score, Model of End-Stage Liver Disease (MELD score), and Barcelona clinic liver cancer (BCLC) staging are used in this assessment.

Serum alpha-fetoprotein was measured in patients and controls and in HCC patients twice: before and 1 month after the intervention. It was determined using a human AFP EIA kit lot. REF 600-10 was manufactured by CanAg Diagnostics AB, Majnabble Terminal SE-414 55 Gothenburg, Sweden.

Serum AREG was measured using amphiregulin (Human) OmniKine sandwich ELISA Kit MBS9502096 (MyBioSource, San Diego, CA, USA) according to manufacturer recommendations. For patients with HCC, serum AREG was measured twice: before and 1 month after the intervention.

Abdominal ultrasonography is for detecting liver cirrhosis, ascites, and hepatic focal lesions.

Triphasic abdominal CT scan was performed in three phases of contrast enhancement (early and late arterial and portal venous phases). It was carried out for any patient showing a suspected focal lesion in the abdominal ultrasound, and follow-up triphasic CT was done in patients with HCC 1 month after the intervention.

Radiofrequency ablation was used for patients with (BCLC-0) or (BCLC-A) HCC, who were ineligible for surgical resection or transplantation, and had tumor diameter below $5 \mathrm{~cm}$ or less than three nodules with $3 \mathrm{~cm}$ as a maximum diameter. RF was performed by inserting an electrode into the lesion generating thermal destruction area encircling the tumor (temperature ranging from 60 to $100{ }^{\circ} \mathrm{C}$ ). When ablated tissues are heated, blood 
vessels nearby convectively cool ablated tissues, generating a heat-sink effect [10].

TACE was used for intermediate BCLC stages patients with Child A-B, large or multifocal HCC who were unsuitable for resection or RF. It was done first by injecting lipiodol emulsion in the tumor feeding artery and then embolization to achieve the synergistic effect of drug cytotoxicity and ischemia [11].

Data were analyzed using the IBM SPSS V18. Descriptive statistics were computed for quantitative data as mean and standard deviation.

Independent $t$ test, paired $t$ test, Mann-Whitney $U$, and Wilcoxon signed-rank tests were used for quantitative data. However, the chi-square and Fisher's exact tests were used for qualitative data.

The significance level was $p$ value $<0.05$ which is significant, and $<0.01$ is highly significant; otherwise is insignificant.

\section{Results}

This study included $60 \mathrm{HCV}$ infected patients: thirty patients with liver cirrhosis-related HCC (their mean age was $57.5 \pm 6.3$ years old (22 males (73.3\%) and 8 females $(26.7 \%)))$ and thirty patients with liver cirrhosis without HCC (their mean age was $54.4 \pm 8.7$ years old (18 males (60\%) and 12 females (40\%))). It was found that 45 patients were Child-Pugh A class (25 in the HCC group and 20 in the cirrhosis group), and 15 patients were Child-Pugh B (5 in the HCC group and 10 in the cirrhosis group). Demographic and laboratory data are shown in Table 1.

In the current study, baseline serum AREG was significantly higher in HCC patients compared with cirrhosis patients $(23.2 \pm 11.5$ vs. $11.1 \pm 7.1)$, with a $p$ value $<0.001$. The HCC group also had a greater AFP $(725.4 \pm 675.2$ vs. $89 \pm 102$ ) (Table 2).

Regarding triphasic CT findings in the HCC group, patients with multiple focal lesions had statistically significantly higher AREG levels than those with a single lesion ( $25.1 \pm 12.5$ vs. $17.2 \pm 4$ ), with a $p$ value of 0.015 . Moreover, it was higher in patients with larger-sized focal lesions $(>3 \mathrm{~cm})$ compared with those with hepatic focal lesions $<3 \mathrm{~cm}(27.9 \pm 12.3$ vs. $16.3 \pm 5.5)$, with a $p$ value of 0.002 . When using Person multivariate correlation, tumor size was the only parameter that significantly correlated with AREG level (Tables 3 and 4).

Patients underwent locoregional treatment according to the approved selection criteria, where 10 patients were candidate for RF and 20 for TACE. Five patients had HCC recurrence 1 month after the intervention (3 with RF and 2 with TACE).

At 1 month follow-up after locoregional treatment, AREG level significantly declined in comparison to its
Table 1 Demographic and laboratory parameters of the studied groups

\begin{tabular}{|c|c|c|c|}
\hline Variables & $\mathrm{HCC}(N=30)$ & Cirrhosis $(N=30)$ & $P$ value \\
\hline \multicolumn{4}{|l|}{ Age (years) } \\
\hline Mean \pm SD & $57.5 \pm 6.3$ & $54.4 \pm 8.7$ & \multirow[t]{2}{*}{$\wedge 0.112$} \\
\hline Range & $46.0-68.0$ & $42.0-68.0$ & \\
\hline \multicolumn{4}{|l|}{ BMI $\left(\mathrm{kg} / \mathrm{m}^{2}\right)$} \\
\hline Mean \pm SD & $24.8 \pm 2.3$ & $24.9 \pm 3.0$ & \multirow[t]{2}{*}{$\wedge 0.885$} \\
\hline Range & $22.0-29.0$ & $20.0-29.0$ & \\
\hline \multicolumn{4}{|l|}{ Gender $(n, \%)$} \\
\hline Male & $22(73.3 \%)$ & $18(60.0 \%)$ & \multirow[t]{2}{*}{${ }^{\#} 0.273$} \\
\hline Female & $8(26.7 \%)$ & $12(40.0 \%)$ & \\
\hline \multicolumn{4}{|c|}{ Child score $(n, \%)$} \\
\hline A & $25(83.3 \%)$ & $20(66.7 \%)$ & \multirow[t]{2}{*}{${ }^{\#} 0.136$} \\
\hline B & $5(16.7 \%)$ & $10(33.3 \%)$ & \\
\hline \multicolumn{4}{|l|}{ MELD score } \\
\hline Mean \pm SD & $7.8 \pm 4.9$ & $7.7 \pm 5.3$ & \multirow[t]{2}{*}{$\wedge 0.967$} \\
\hline Range & $1.0-18.3$ & $1.0-20.1$ & \\
\hline \multicolumn{4}{|c|}{ Hemoglobin (g/dL) } \\
\hline Mean \pm SD & $12.0 \pm 1.6$ & $12.3 \pm 1.0$ & \multirow[t]{2}{*}{$\wedge 0.429$} \\
\hline Range & $9.5-14.0$ & $10.8-14.2$ & \\
\hline \multicolumn{4}{|l|}{$\operatorname{TLC}\left(\times 10^{3} / \mathrm{mL}\right)$} \\
\hline Mean \pm SD & $6.7 \pm 2.0$ & $6.6 \pm 1.4$ & \multirow[t]{2}{*}{$\wedge 0.754$} \\
\hline Range & $3.8-9.7$ & $4.7-9.3$ & \\
\hline \multicolumn{4}{|c|}{ Platelets (x $\left.10^{3} / \mathrm{mL}\right)$} \\
\hline Mean \pm SD & $173.2 \pm 38.4$ & $175.9 \pm 44.3$ & \multirow[t]{2}{*}{$\wedge 0.802$} \\
\hline Range & $89.0-258.0$ & $112.0-246.0$ & \\
\hline \multicolumn{4}{|l|}{ AST (IU/L) } \\
\hline Mean \pm SD & $50.9 \pm 28.3$ & $30.8 \pm 14.8$ & \multirow[t]{2}{*}{$\wedge 0.001^{*}$} \\
\hline Range & $16.0-111.0$ & $12.0-88.0$ & \\
\hline \multicolumn{4}{|l|}{ ALT (IU/L) } \\
\hline Mean \pm SD & $60.5 \pm 32.5$ & $36.4 \pm 17.1$ & \multirow[t]{2}{*}{$\wedge 0.001 *$} \\
\hline Range & $11.0-132.0$ & $18.0-76.0$ & \\
\hline \multicolumn{4}{|l|}{ Albumin (g/dL) } \\
\hline Mean \pm SD & $3.7 \pm 0.4$ & $3.8 \pm 0.4$ & \multirow[t]{2}{*}{$\wedge 0.111$} \\
\hline Range & $2.9-4.5$ & $3.0-4.7$ & \\
\hline \multicolumn{4}{|c|}{ Total bilirubin (mg/dL) } \\
\hline Mean \pm SD & $1.3 \pm 0.5$ & $1.4 \pm 0.5$ & \multirow[t]{2}{*}{$\wedge 0.303$} \\
\hline Range & $0.4-2.1$ & $0.3-2.3$ & \\
\hline Direct bilirubir & $g / d L)$ & & \\
\hline Mean \pm SD & $0.6 \pm 0.2$ & $0.7 \pm 0.3$ & $\wedge 0.165$ \\
\hline Range & $0.1-1.1$ & $0.1-1.1$ & \\
\hline Alkaline Ph. (m & & & \\
\hline Mean \pm SD & $76.6 \pm 25.0$ & $81.8 \pm 29.1$ & $\wedge 0.458$ \\
\hline Range & $40.0-138.0$ & $44.0-121.0$ & \\
\hline INR & & & \\
\hline Mean \pm SD & $76.6 \pm 25.0$ & $81.8 \pm 29.1$ & $\wedge 0.111$ \\
\hline Range & $40.0-138.0$ & $44.0-121.0$ & \\
\hline
\end{tabular}

$\wedge$ Independent $t$ test

\# Chi square test

*Significant 
Table 2 Comparison between the two studied groups regarding baseline amphiregulin and AFP

\begin{tabular}{|c|c|c|c|}
\hline Variables & $\mathrm{HCC}(N=30)$ & Cirrhosis $(N=30)$ & $\wedge P$ value \\
\hline \multicolumn{4}{|l|}{$\operatorname{AFP}(\mathrm{ng} / \mathrm{mL})$} \\
\hline Mean \pm SD & $725.4 \pm 675.2$ & $89.0 \pm 102.0$ & $<0.001^{*}$ \\
\hline Range & $110.0-2243.0$ & $9.0-356.0$ & \\
\hline \multicolumn{4}{|c|}{ Amphiregulin (pg/mL) } \\
\hline Mean \pm SD & $23.2 \pm 11.5$ & $11.1 \pm 7.1$ & $<0.001^{*}$ \\
\hline Range & $6.4-49.7$ & $1.4-29.0$ & \\
\hline
\end{tabular}

$\wedge$ Independent $t$ test

*Significant

Table 3 Comparison between baseline amphiregulin serum level $(\mathrm{pg} / \mathrm{mL})$ and other studied parameters among the HCC group

\begin{tabular}{|c|c|c|c|}
\hline \multirow[t]{2}{*}{ HCC group } & \multicolumn{2}{|c|}{ Amphiregulin (pg/mL) } & \multirow[t]{2}{*}{$P$ value } \\
\hline & $N$ & Mean \pm SD & \\
\hline \multicolumn{4}{|l|}{ Gender } \\
\hline Male & 22 & $23.1 \pm 9.0$ & $\wedge 0.950$ \\
\hline Female & 8 & $23.6 \pm 17.5$ & \\
\hline \multicolumn{4}{|l|}{ Child score } \\
\hline A & 25 & $23.7 \pm 12.1$ & $\wedge 0.636$ \\
\hline B & 5 & $21.0 \pm 8.8$ & \\
\hline \multicolumn{4}{|l|}{ Foci number } \\
\hline Solitary & 7 & $17.2 \pm 4.0$ & $\wedge 0.015^{*}$ \\
\hline Multiple & 23 & $25.1 \pm 12.5$ & \\
\hline \multicolumn{4}{|c|}{ Focal lesion size } \\
\hline$\leq 3 \mathrm{~cm}$ & 12 & $16.3 \pm 5.5$ & $\wedge 0.002 *$ \\
\hline$>3 \mathrm{~cm}$ & 18 & $27.9 \pm 12.3$ & \\
\hline \multicolumn{4}{|l|}{ BCLC } \\
\hline A & 6 & $19.3 \pm 5.0$ & \#0.654 \\
\hline B & 21 & $24.2 \pm 12.3$ & \\
\hline C & 3 & $24.5 \pm 17.4$ & \\
\hline \multicolumn{4}{|l|}{ Intervention } \\
\hline TACE & 20 & $22.5 \pm 12.4$ & $\wedge 0.603$ \\
\hline RF & 10 & $24.8 \pm 10.2$ & \\
\hline
\end{tabular}

$\wedge$ Independent $t$ test

\# ANOVA test

*Significant

baseline levels (from $23.2 \pm 11.5$ to $19.4 \pm 10.9$ ) with overall $p$ value 0.012 , while follow-up levels of AFP showed insignificant reduction (from $725.4 \pm 675.2$ to $717.3 \pm 732.9$ ) with $p$ value 0.953 (Table 5).

Three patients were found to have a branch portal vein thrombus with a patent main portal vein among HCC patients; those patients received TACE as a locoregional therapy.
Table 4 Correlations of AFP and amphiregulin with other parameters among HCC group

\begin{tabular}{llllll}
\hline Variables & \multicolumn{2}{l}{ AFP } & & \multicolumn{2}{l}{ Amphiregulin } \\
\cline { 2 - 3 } \cline { 5 - 6 } & $\boldsymbol{r}$ & $\boldsymbol{p}$ & & $\boldsymbol{r}$ & $\boldsymbol{p}$ \\
\hline Age & 0.123 & 0.518 & & -0.018 & 0.925 \\
Body mass index & 0.166 & 0.382 & & -0.068 & 0.720 \\
Child score & -0.128 & 0.500 & & -0.118 & 0.533 \\
Tumor size & 0.079 & 0.676 & & 0.637 & $<0.001^{*}$ \\
Hemoglobin & 0.008 & 0.965 & & -0.162 & 0.392 \\
TLC & 0.021 & 0.914 & & -0.266 & 0.155 \\
Platelets & -0.026 & 0.893 & & -0.098 & 0.606 \\
AST & 0.080 & 0.673 & & -0.007 & 0.972 \\
ALT & 0.111 & 0.561 & & 0.213 & 0.259 \\
Albumin & 0.088 & 0.644 & & 0.021 & 0.913 \\
Total bilirubin & -0.079 & 0.679 & & -0.129 & 0.498 \\
Direct bilirubin & -0.097 & 0.611 & -0.156 & 0.409 \\
Alkaline Ph. & 0.186 & 0.324 & 0.059 & 0.757 \\
INR & -0.265 & 0.156 & -0.019 & 0.919 \\
Creatinine & 0.177 & 0.349 & -0.241 & 0.199 \\
MELD Score & -0.058 & 0.759 & -0.229 & 0.223 \\
Amphiregulin & 0.080 & 0.673 & & \\
\hline Pearson correlation & & & & \\
*Significant & & & &
\end{tabular}

Table 5 Comparison between serum amphiregulin and AFP levels before and 1 month after intervention among HCC group

\begin{tabular}{llll}
\hline Time & Measures & AFP $(\mathrm{ng} / \mathrm{mL})$ & $\begin{array}{l}\text { Amphiregulin } \\
(\mathbf{p g} / \mathrm{mL})\end{array}$ \\
\hline Before & Mean \pm SD & $725.4 \pm 675.2$ & $23.2 \pm 11.5$ \\
& Range & $110.0-2243.0$ & $6.4-49.7$ \\
After & Mean \pm SD & $717.3 \pm 732.9$ & $19.4 \pm 10.9$ \\
& Range & $100.0-2113.0$ & $1.7-50.9$ \\
$\wedge$ Pvalue & & 0.953 & $\mathbf{0 . 0 1 2}$ \\
\hline
\end{tabular}

Cl Confidence interval

$\wedge$ Paired $t$ test

*Significant

The BCLC class B had the highest cure rate (80\%), whereas $\mathrm{BCLC}$ class $\mathrm{C}$ had the highest recurrence rate (60\%) for HCC (Table 6).

In terms of laboratory data, serum albumin and hemoglobin levels were significantly lower among patients who had a recurrence of HCC, while other parameters were statistically insignificant, as shown in Table 7.

At follow-up, the serum AREG level was statistically significantly higher in recurrence cases than in remission ones $(30.8 \pm 14.1$ vs. $17.2 \pm 8.8)$, with a $p$ value of 0.008 . The same was found for the AFP level (1343.4 $\pm 907.3 \mathrm{vs}$. $592 \pm 643.3$ ), with a $p$ value of 0.034 (Table 8). 
Table 6 Comparison according to tumor recurrence among HCC group regarding tumor characteristics

\begin{tabular}{|c|c|c|c|}
\hline Variables & Recurrence $(N=5)$ & Remission $(N=25)$ & $P$ value \\
\hline Size $\left(\mathrm{cm}^{2}\right)$ & $3.0 \pm 2.8$ & $4.0 \pm 1.5$ & $\wedge 0.265$ \\
\hline \multicolumn{4}{|l|}{ Number } \\
\hline Solitary & $2(40.0 \%)$ & $5(20.0 \%)$ & \multirow[t]{2}{*}{${ }^{\#} 0.565$} \\
\hline Multiple & $3(60.0 \%)$ & $20(80.0 \%)$ & \\
\hline \multicolumn{4}{|l|}{ BCLC } \\
\hline A & 1 (20.0\%)a & 5 (20.0\%)a & \multirow[t]{3}{*}{${ }^{\#} 0.002^{*}$} \\
\hline B & 1 (20.0\%)a & $20(80.0 \%) b$ & \\
\hline C & $3(60.0 \%) a$ & $0(0.0 \%) \mathrm{b}$ & \\
\hline \multicolumn{4}{|l|}{ Intervention } \\
\hline TACE & $2(40.0 \%)$ & $18(72.0 \%)$ & \multirow[t]{2}{*}{ \#0.300 } \\
\hline RF & $3(60.0)$ & $7(28.0 \%)$ & \\
\hline
\end{tabular}

$\wedge$ Independent $t$ test

\# Fisher's exact test with post hoc test

*Significant

ROC curve was used to reveal the diagnostic effect of AREG and AFP levels to determine the appropriate cutoff value for distinguishing HCC from Cirrhosis (Table 9 and Fig. 1).

The optimal cutoff value for predicting HCC recurrence was identified using a $\mathrm{ROC}$ curve for baseline AREG and AFP (Table 10 and Fig. 2).

Another ROC curve was performed for 1 month follow-up AREG and AFP to get the best cutoff value to predict tumor recurrence in HCC patients (Table 11, Fig. 3).

\section{Discussion}

Hepatocellular carcinoma is the fifth most prevalent malignancy worldwide, and its prevalence is rising. The presence of liver cirrhosis is the major risk factor caused primarily by chronic HCV and HBV infection [12].
Chronic HCV infection is a potent pro-inflammatory factor, promoting hepatocarcinogenesis. Accordingly, resolution of $\mathrm{HCV}$ infection should result in a reduced incidence of $\mathrm{HCC}$, even in patients with liver cirrhosis [13].

In general, $\mathrm{HCC}$ patients have a poor prognosis because they are mostly diagnosed at advanced stages. Regular screening programs among high-risk populations could lead to an earlier diagnosis of HCC [14].

Early detection of patients with $\mathrm{HCC}$ is needed because it gives a better prognosis as HCC tends to grow slowly and stay confined to the liver. Early HCC detection is attainable employing ultrasonic scanning and AFP measurement, although using AFP as a screening test is confounded by numerous false positives and negatives; so, if imaging and tumor markers were combined, early diagnosis of HCC would not be difficult [14].

AFP has been widely used for HCC diagnosis and follow-up. However, it is not always elevated to a diagnostic level in all patients, particularly in small HCC; thus, adding other diagnostic tools is mandatory [15].

Table 8 Comparison according to tumor recurrence among HCC group regarding amphiregulin and AFP levels

\begin{tabular}{|c|c|c|c|}
\hline Time & Recurrence $(N=5)$ & Remission $(N=25)$ & $\wedge P$ value \\
\hline \multicolumn{4}{|l|}{ AFP (ng/mL) } \\
\hline Baseline & $565.0 \pm 207.7$ & $757.5 \pm 733.0$ & 0.278 \\
\hline Follow-up & $1343.4 \pm 907.3$ & $592.0 \pm 643.3$ & $0.034^{*}$ \\
\hline \multicolumn{4}{|c|}{ Amphiregulin (pg/mL) } \\
\hline Baseline & $24.5 \pm 12.4$ & $23.0 \pm 11.6$ & 0.798 \\
\hline Follow-up & $30.8 \pm 14.1$ & $17.2 \pm 8.8$ & $0.008^{*}$ \\
\hline
\end{tabular}

$\wedge$ Independent $t$ test

*Significant

Table 7 Comparison according to tumor recurrence among HCC group regarding baseline laboratory results

\begin{tabular}{|c|c|c|c|}
\hline Variables & Recurrence $(N=5)$ & Remission $(N=25)$ & $\wedge P$ value \\
\hline Hemoglobin (g/dL) & $10.3 \pm 1.0$ & $12.4 \pm 1.5$ & $0.007^{*}$ \\
\hline $\operatorname{TLC}\left(\times 10^{3} / \mathrm{mL}\right)$ & $6.4 \pm 2.3$ & $6.8 \pm 1.9$ & 0.664 \\
\hline Platelets (x 103/mL) & $185.6 \pm 24.4$ & $170.8 \pm 40.5$ & 0.440 \\
\hline AST (IU/L) & $44.2 \pm 31.2$ & $52.2 \pm 28.2$ & 0.572 \\
\hline ALT (IU/L) & $85.0 \pm 47.4$ & $55.6 \pm 27.4$ & 0.063 \\
\hline Albumin (g/dL) & $3.3 \pm 0.2$ & $3.7 \pm 0.4$ & $0.006^{*}$ \\
\hline Total bilirubin (mg/dL) & $1.4 \pm 0.6$ & $1.3 \pm 0.5$ & 0.525 \\
\hline Direct bilirubin (mg/dL) & $0.7 \pm 0.2$ & $0.6 \pm 0.3$ & 0.295 \\
\hline Alkaline Ph. (mg/dL) & $72.0 \pm 21.5$ & $77.5 \pm 26.0$ & 0.663 \\
\hline INR & $1.5 \pm 0.5$ & $1.2 \pm 0.4$ & 0.135 \\
\hline Creatinine (mg/dL) & $1.00 \pm 0.37$ & $0.80 \pm 0.34$ & 0.261 \\
\hline
\end{tabular}

$\wedge$ Independent $t$ test

*Significant 
Table 9 Diagnostic performance of AFP and amphiregulin in differentiating HCC group from cirrhosis group

\begin{tabular}{llllllrr}
\hline Marker & AUC & Sensitivity (\%) & Specificity (\%) & SE & $P$ & 95\% Cl & Cutoff \\
\hline AFP & 0.952 & 86.7 & 90 & 0.024 & $<0.001^{*}$ & $0.906-0.999$ & $\geq 220.0$ \\
Amphiregulin & 0.817 & 63.3 & 86.7 & 0.053 & $<0.001^{*}$ & $0.713-0.922$ & $\geq 17.0$ \\
\hline
\end{tabular}

AUC Area under curve, SE Standard error, CI Confidence interval

*Significant

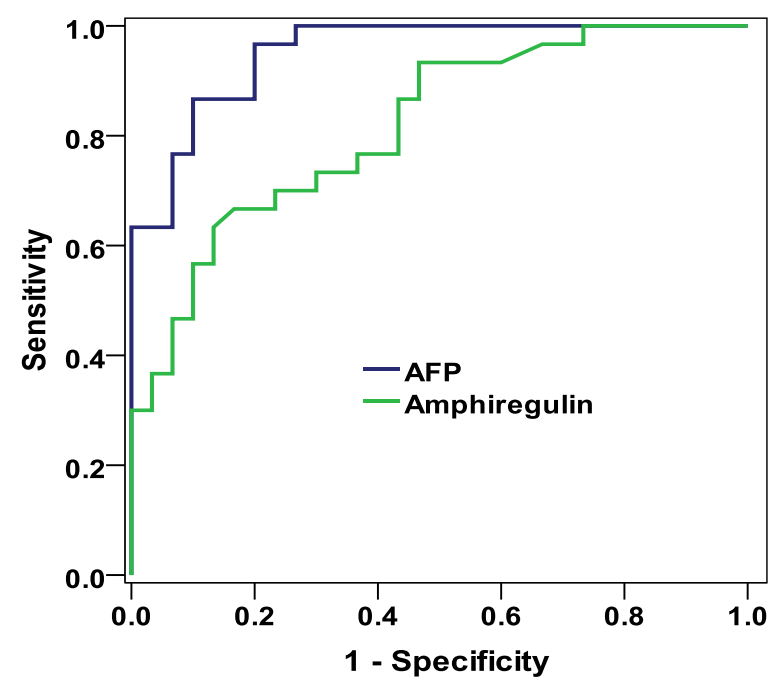

Fig. 1 ROC curve for AFP and amphiregulin in differentiating HCC from cirrhosis

The benefit of using AFP in combination with ultrasonography is also debated, including discrepant recommendations in guidelines. The American Association for the Study of Liver Diseases recommends using ultrasonography with or without AFP, leaving it up to the clinician to consider the benefits and drawbacks in each patient. In contrast, European guidelines recommend ultrasonography alone. In an attempt to increase the performance, the statistical model GALAD incorporates AFP levels, the biomarkers AFP-L3 percentage, desgamma-carboxy prothrombin, and the sex and age of the patient into one model [16].

The study aims to evaluate the diagnostic role of amphiregulin as a possible marker for HCC and its prognostic role in detecting tumor recurrence after different locoregional therapeutic modalities.
This study was done on 60 patients divided into 2 groups: the HCC group including 30 cirrhotic patients with HCC who underwent either RF or TACE and the cirrhosis group including 30 randomly selected cirrhotic patients as control.

In this study, HCC was more prevalent in men (22; $73.3 \%)$ than in women $(8 ; 26.7 \%)$. These results are consistent with those of a previous study, showing a higher incidence of HCC among males [17]. However, they were contrary to what was found by Tokushige et al. [18]. This may be explained in part by the differences in sample size, sex hormones, and risk factors exposure. It has been proposed that sex hormones, iron deposition, and ethnic differences could all influence hepatocarcinogenesis, explaining why HCC is more prevalent in men [19].

In the current study, the ages of patients with HCC ranged between 46 and 68 years with a mean $57.5 \pm 6.3$ years which is likely dependent on how long the liver has been diseased. Those findings agreed with Konstantin et al. [20] findings that age of HCC patients was $63.79 \pm 9.99$ years. Those findings were also consistent with those of Oliver et al. [21] who stated that age of HCC patients was $59.7 \pm 10.4$ years. This validates the prevalence of $\mathrm{HCC}$ in the fifth and sixth decades of life. This is older than age of liver cirrhosis patients which ranged from 43 to 65 with a mean of $54.8 \pm 7.6$ which was in agreement with Konstantin et al. [20].

The current work revealed no significant difference in Child and MELD scores between patients with cirrhosis and those with HCC. Further, $83.3 \%$ of patients with $\mathrm{HCC}$ were found to be Child A, which agreed with D'Amico et al. [22], who stated that HCC could develop at any stage of cirrhosis.

Moreover, there is no difference between the studied groups regarding complete blood picture and full hepatic profile, except for AST and ALT levels, which were statistically higher in HCC patients than in those with

Table 10 Diagnostic performance of baseline AFP and amphiregulin in predicting tumor recurrence

\begin{tabular}{|c|c|c|c|c|c|c|c|}
\hline Marker & AUC & Sensitivity (\%) & Specificity (\%) & SE & $P$ & $95 \% \mathrm{Cl}$ & Cutoff \\
\hline Baseline AFP & 0.632 & 80 & 56 & 0.095 & 0.359 & $0.445-0.819$ & $\geq 439.0$ \\
\hline Baseline amphiregulin & 0.552 & 80 & 40 & 0.137 & 0.718 & $0.284-0.820$ & $\geq 17.0$ \\
\hline
\end{tabular}




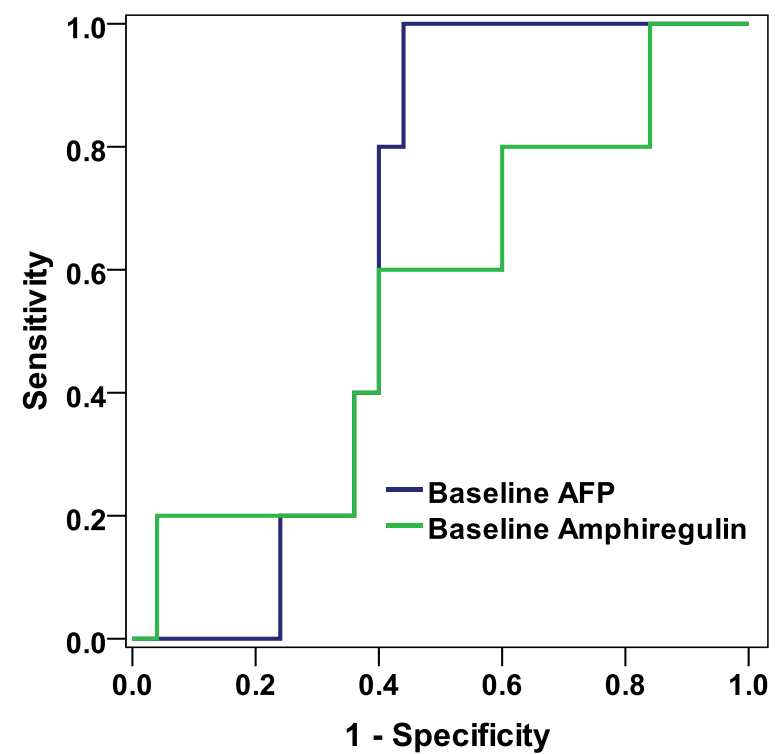

Fig. 2 ROC curve for baseline AFP and amphiregulin in predicting tumor recurrence

cirrhosis. These results could be explained by Baghdady et al. [23], stating that the elevated aminotransferases value in HCC reflects damage to adjacent hepatocytes as a direct result of tumor growth or damage to more remote liver cells caused by interference with their blood supply or venous drainage. It may also be due to continuing liver cell necrosis in those with concomitant active cirrhosis or chronic active hepatitis.

It has been found that HCC patients had statistically significant higher mean serum AREG values than cirrhotic patients $(23.2 \mathrm{pg} / \mathrm{ml}$ and $11.1 \mathrm{pg} / \mathrm{ml}$, respectively) which is consistent with Awad et al. [24].

Those results revealed a worthy positive correlation between AREG level and hepatocellular carcinoma, which were similar to those of Han et al. [25], showing that AREG levels were upregulated in patients with HCC and could be a possible diagnostic biomarker for HCC.

This study discovered a link between AREG levels and progression of $\mathrm{HCC}$, demonstrating that increased AREG levels were associated with increasing tumor size or the number of lesions. Although these results were not previously studied upon HCC, they were compatible with Watanabe et al. [26] and Yamada et al. [27], reporting that

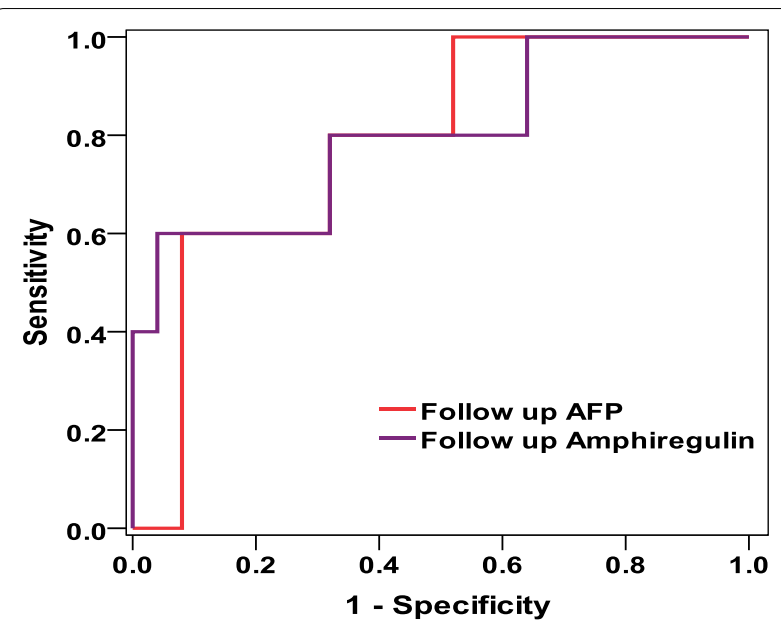

Fig. 3 ROC curve for follow-up AFP and amphiregulin in diagnosing and predicting tumor recurrence

AREG overexpression was associated with liver metastasis in primary colorectal cancer (CRC). Kuramochi et al. [28] also discovered a link between AREG expression in metastatic liver tumors and primary CRC. Further, Chayangsu et al. [29] showed that advanced CRC cases had higher serum AREG levels.

The current study found that AREG levels declined significantly 1 month after intervention, with a much greater drop in patients treated with chemoembolization than with radiofrequency. It could be explained by the fact that patients with BCLC (B) who received TACE had significantly greater levels of AREG than those with BCLC (A) who underwent RF.

These findings are consistent with those of Han et al. [25], who discovered that the AREG level in HCC patients had significantly decreased after the intervention, indicating that AREG levels correlated with cure and implying the importance of monitoring HCC patients after the intervention.

On the other hand, the present study revealed that AFP levels did not decrease significantly 1 month following treatment regardless of whether TACE or RF was used. These findings go against those of Corey and Pratts [30], reporting a link between AFP and HCC severity. These contradictory results may be explained by Johnson's finding [31] that only $80.3 \%$ of patients experienced a decline in AFP within 30 days after effective curative invasive

Table 11 Diagnostic performance of follow-up AFP and amphiregulin in diagnosing tumor recurrence

\begin{tabular}{llllllll}
\hline Marker & AUC & Sensitivity (\%) & Specificity (\%) & SE & $\boldsymbol{P}$ & $\mathbf{9 5 \% C l}$ & Cutoff \\
\hline Follow-up AFP & 0.784 & 60 & 92 & 0.100 & $\mathbf{0 . 0 4 8}^{*}$ & $0.588-0.980$ & $\geq 1993.0$ \\
Follow-up amphiregulin & 0.800 & 60 & 96 & 0.120 & $\mathbf{0 . 0 3 7}^{*}$ & $0.464-1.000$ & $\geq 29.7$ \\
\hline
\end{tabular}


interventions. It could be attributed to micro-metastatic lesions or residual cancer cells derived from HCC colonies in blood circulation.

This conflict could be attributed to different sample sizes, study populations, HCC characteristics, and variable follow-up periods.

According to the current study, five patients (16.7\%) had a recurrence of their $\mathrm{HCC}$ after the intervention, with a statistically significant higher age than those who went into remission. BCLC grade-C was significantly more frequent in recurrence cases, and none of the patients with BCLC $(\mathrm{C})$ had remission. These findings are consistent with those of $\mathrm{Xu}$ et al. [32], indicating that BCLC-B/C HCC patients have a poor prognosis, which may be attributed to intrahepatic recurrence caused by intrahepatic metastasis or multicentric new tumors being distinguishable from their different time to tumor recurrence.

Further, significantly lower hemoglobin and albumin levels were found in recurrent $\mathrm{HCC}$ patients than in those in remission. These findings corroborate those of a previous study, revealing that decreased albumin levels are linked to enhanced HCC aggressiveness parameters and their involvement in promoting systemic inflammation, which has been associated with poor prognosis [33].

The follow-up AFP was statistically significantly higher in recurrence cases than in remission ones, which agrees with a previous study [30], reporting that AFP level could track how well a patient responds to treatment.

At follow-up, AREG was statistically significantly higher in recurrence cases in comparison to cases in remission; this may be due to overexpression of AREG by hepatocellular carcinoma cells as described by Castillo et al. [34] who stated that HCC cells overexpress and produce AREG, leading to an autocrine stimulation loop to achieve self-sufficiency in growth signals. These findings corroborate those of Bai et al. [35], who found that AREG levels were elevated in HCC patients and might be employed as a possible biomarker for HCC diagnosis.

The accuracy of AFP for detecting HCC varies according to the cutoff parameters employed. According to the current findings, at a cutoff of $\geq 220 \mathrm{ng} / \mathrm{ml}$, the sensitivity, specificity, accuracy, positive predictive value (PPV), and negative predictive value (NPV) were $86.7 .0 \%, 90 \%$, $88.3 \%, 89.7 .0 \%$, and $87.1 \%$, respectively.

These findings are similar to those of Debruyne and Delanghe [36], who found that when the cutoff value is $20 \mathrm{ng} / \mathrm{ml}$, serum AFP has a $65 \%$ sensitivity and $94 \%$ specificity. The current findings were not comparable to those of Clark [37], who found that AFP has poor sensitivity even at low cutoffs (e.g., $20 \mathrm{ng} / \mathrm{mL}$, sensitivity $64.3 \%$ ) and is considerably more limited in smaller tumors $(25 \%$ sensitivity at tumor size $3 \mathrm{~cm})$. The race,
HCC etiology, and hepatic inflammation intensity are all linked to an increase in AFP in patients with cirrhosis with or without HCC, according to Yang et al. [38].

On the other hand, the serum AREG level cutoff value of $\geq 17 \mathrm{pg} / \mathrm{ml}$ exhibited a $63.3 \%$ sensitivity, $86.7 \%$ specificity, $75 \%$ accuracy, $82.6 \% \mathrm{PPV}$, and $70.3 \% \mathrm{NPV}$ in predicting HCC.

Subsequently, it could be concluded that compared with AFP, AREG had moderate significant diagnostic performance in distinguishing $\mathrm{HCC}$ from liver cirrhosis patients.

In the current study, baseline AREG and AFP levels had no substantial diagnostic performance in predicting tumor recurrence, which is consistent with previous studies [20,39], revealing no difference between patients with and without $\mathrm{HCC}$ recurrence regarding baseline AFP levels.

However, follow-up of AREG and AFP levels after 1 month was significantly diagnostic for tumor recurrence, with AREG being more significant. These findings are consistent with those of Yu et al. [40], reporting that measuring serial AFP levels to assess biological response is a useful predictor of overall survival.

At a cutoff value of $\geq 29.7 \mathrm{pg} / \mathrm{ml}$, AREG was found to have $60 \%$ sensitivity, $96 \%$ specificity, $90 \%$ accuracy, 75\% PPV, and $92.3 \% \mathrm{NPV}$ in predicting possible HCC recurrence.

There are limitations in this study that could be considered in future research, such as the small number of analyzed populations and the need for a longer followup period to detect further tumor recurrence.

A greater number of patients and follow-up over several years is recommended to assess AREG role in detecting recurrence of $\mathrm{HCC}$ after successful therapy. More studies about AREG in liver metastasis are also needed.

\section{Conclusions}

AREG may be considered a possible diagnostic marker for HCC. Further, it can be employed as a prognostic marker for HCC after treatment, as its follow-up level was useful in predicting possible tumor recurrence.

\footnotetext{
Acknowledgements

The authors are grateful to the staff members of Ain Shams University's Clinical Pathology, Interventional Radiology, and Gastroenterology and Hepatology departments.

\section{Authors' contributions}

Aside from being the corresponding author, Al suggested the research topic, completed a final review of the collected data, and contributed to the manuscript preparation. SMM assisted in the collection of data. OAA made a significant contribution to the manuscript's writing and critical revision. AGM helped in collecting and revising the data. HSR contributed to the interpretation of all statistical analyses of the obtained data. The submitted manuscript was revised and approved by all authors.
} 


\section{Funding}

There was no specific grant for this research from any funding agencies.

\section{Availability of data and materials}

On reasonable request, the corresponding author will provide the data used and analyzed during the current work.

\section{Declarations}

\section{Ethics approval and consent to participate}

This study was in accordance with the ethical principles of the 1975 Helsink Declaration that was granted by the local Ethics Committee of Ain Shams University Faculty of medicine (FWA 000017585) December 2018. Participants gave their informed written consent before being enrolled in the trial, after being informed about the study's goal and required interventions.

\section{Consent for publication}

Participants provided written permission for the study's findings to be published

\section{Competing interests}

There are no competing interests declared by the authors.

Received: 10 July 2021 Accepted: 17 October 2021

Published online: 08 November 2021

\section{References}

1. Cucchetti A, Trevisani F, Bucci L et al (2016) Years of life that could be saved from prevention of hepatocellular carcinoma. Aliment Pharmacol Ther 43(7):814-824

2. Welzel TM, Graubard BI, Zeuzem S et al (2011) Metabolic syndrome increases the risk of primary liver cancer in the United States: a study in the SEER-medicare database. Hepatology 54(2):463-471

3. Zhang ZQ, Meng H, Wang N et al (2014) Serum microRNA 143 and microRNA 215 as potential biomarkers for the diagnosis of chronic hepatitis and hepatocellular carcinoma. Diagn Pathol 9:135

4. Cho YK, Kim JK, Kim MY et al (2009) Systematic review of randomized trials for hepatocellular carcinoma treated with percutaneous ablation therapies. Hepatology 49(2):453-459

5. Xie ZB, Wang XB, Peng YC et al (2015) Systematic review comparing the safety and efficacy of conventional and drug-eluting bead transarterial chemoembolization for inoperable hepatocellular carcinoma. Hepatol Res 45:190-200

6. Hung YC, Lin CL, Liu CJ et al (2015) Development of risk scoring system for stratifying population for hepatocellular carcinoma screening. Hepatology 61:1934-1944

7. Vilchez V, Turcios L, Marti F et al (2016) Targeting Wnt/ $\beta$-catenin pathway in hepatocellular carcinoma treatment. World J Gastroenterol 22(2):823-832

8. Berasain C, Avila MA (2014) Amphiregulin. Semin Cell Dev Biol 28:31-41. https://doi.org/10.1016/j.semcdb.2014.01.005

9. Bruix J, Sherman M (2011) Management of hepatocellular carcinoma: an update. Hepatology 53(3):1020-1022. https://doi.org/10.1002/hep.24199

10. Poulou LS, Botsa E, Thanou I et al (2015) Percutaneous microwave ablation vs radiofrequency ablation in the treatment of hepatocellular carcinoma. World J Hepatol 7(8):1054-1063

11. Facciorusso A (2018) Drug-eluting beads transarterial chemoembolization for hepatocellular carcinoma: current state of the art. World J Gastroenterol 24(2):161-169. https://doi.org/10.3748/wjg.v24.i2.161

12. Heimbach JK, Kulik LM, Finn RS et al (2018) AASLD guidelines for the treatment of hepatocellular carcinoma. Hepatology 67(1):358-380

13. El Kassas M, Funk AL, Salaheldin M et al (2018) Increased recurrence rates of hepatocellular carcinoma after DAA therapy in a hepatitis C-infected Egyptian cohort: a comparative analysis. J Viral Hepat 25:623-630

14. Smith R (1997) Screening fundamentals. J Natl Cancer Inst Monogr 1997:15-19
15. Stefaniuk P, Cianciara J, Wiercinska-Drapalo A (2010) Present and future possibilities for early diagnosis of hepatocellular carcinoma. World J Gastroenterol 16(4):418-424

16. Frenette CT, Isaacson AJ, Bargellini I et al (2019) A practical guideline for hepatocellular carcinoma screening in patients at risk. Mayo Clin Proc Innov Qual Outcomes 3:302-310

17. El-Zayadi AR, Badran HM, Barakat EM et al (2005) Hepatocellular carcinoma in Egypt: a single center study over a decade. World J Gastroenterol 11(33):5193-5198

18. Tokushige K, Hyogo H, Nakajima T et al (2016) Hepatocellular carcinoma in Japanese patients with nonalcoholic fatty liver disease and alcoholic liver disease: multicenter survey. J Gastroenterol 51(6):586-596

19. Nishida N, Arizumi T, Hayaishi S et al (2012) Gender differences in the livers of patients with hepatocellular carcinoma and chronic hepatitis C infection. Dig Dis 30(6):547-553

20. Konstantin K, Rode A, Simonsen K et al (2016) Macrophage activation marker soluble CD163 may predict disease progression in hepatocellular carcinoma. Scand J Clin Lab Invest 76(1):64-73

21. Oliver W, Koberle V, Bettinger D et al (2013) Diagnostic and prognostic significance of cell death and macrophage activation markers in patients with hepatocellular carcinoma. J Hepatol 59:769-779

22. D'Amico G, Garcia-Tsao G, Pagiaro L (2006) Natural history and prognostic indications of survival in cirrhosis: a systemic review of 118 studies. J Hepatol 44:217-231

23. Baghdady I, Fouad F, Sayed M et al (2014) Serum markers for the early detection of hepatocellular carcinoma in patients with chronic viral hepatitis C infection. Menoufia Med J 27:544-550

24. Awad AE, Ebrahim MA, Eissa LA et al (2019) Dickkopf-1 and amphiregulin as novel biomarkers and potential therapeutic targets in hepatocellular carcinoma. IJHOSCR 13(3):153-163

25. Han SX, Bai E, Jin GH et al (2014) Expression and clinical significance of YAP, TAZ, and AREG in hepatocellular carcinoma. J Immunol Res 2014:261365

26. Watanabe T, Kobunai T, Yamamoto Y et al (2010) Prediction of liver metastasis after colorectal cancer using reverse transcription-polymerase chain reaction analysis of 10 genes. Eur J Cancer 46(11):2119-2126

27. Yamada M, Ichikawa Y, Yamagishi S et al (2008) Amphiregulin is a promising prognostic marker for liver metastases of colorectal cancer. Clin Cancer Res 14(8):2351-2356

28. Kuramochi H, Nakajima G, Kaneko Y et al (2012) Amphiregulin and epiregulin mRNA expression in primary colorectal cancer and corresponding liver metastases. BMC Cancer 12:88. https://doi.org/10.1186/ 1471-2407-12-88

29. Chayangsu C, Khunsri S, Sriuranpong V et al (2017) The correlations between serum amphiregulin and other clinicopathological factors in colorectal cancer. J Gastrointest Oncol 8(6):980-984

30. Corey KE, Pratts DS (2009) Current status of therapy for hepatocellular carcinoma. Therapy Adv Gastroenterol 2:45-57

31. Johnson PJ (2001) The role of serum alpha-fetoprotein estimation in the diagnosis and management of hepatocellular carcinoma. Clin Liver Dis 5(1):145-159

32. Xu W, Guo R, Xu G et al (2017) Management of intrahepatic recurrence after resection for hepatocellular carcinoma exceeding the barcelona clinic liver cancer criteria. Oncotarget 8(66):110406-110414. https://doi. org/10.18632/oncotarget.22779

33. Carr BI, Guerra V (2017) Serum albumin levels in relation to tumor parameters in hepatocellular carcinoma patients. Int J Biol Mark 32(4):391-396. https://doi.org/10.5301/ijbm.5000300

34. Castillo J, Erroba E, Perugorría MJ et al (2006) Amphiregulin contributes to the transformed phenotype of human hepatocellular carcinoma cells. 66(12):6129-6138

35. Bai DS, Zhang C, Chen P et al (2017) The prognostic correlation of AFP level at diagnosis with pathological grade, progression, and survival of patients with hepatocellular carcinoma. Sci Rep 7(1):12870

36. Debruyne EN, Delanghe JR (2008) Diagnosing and monitoring hepatocelIular carcinoma with alpha-fetoprotein: new aspects and applications. Clin Chim Acta 395:19-26. https://doi.org/10.1016/j.cca.2008.05.010

37. Clark P (2016) Serum markers for hepatocellular carcinoma. Clin Liver Dis 8(2):29-33

38. Yang JD, Dai J, Singal AG et al (2017) Improved performance of serum alpha-fetoprotein for hepatocellular carcinoma diagnosis in HCV cirrhosis 
with normal alanine transaminase. Cancer Epidemiol Prev Biomarkers 26(7):1085-1092

39. Adaninggar PN, Kholili U, Maimunah U et al (2016) Response evaluation of patients undergoing transarterial chemoembolization (TACE) for hepatocellular carcinoma with respect to tumour size, number of lesion and alpha-fetoprotein (AFP) level. Indones I Gastroenterol Hepatol Dig Endosc 17(1):3-9

40. Yu SJ, Kwon JH, Kim W et al (2018) Initial al pha-fetoprotein response predicts prognosis in hepatitis B-related solitary HCC patients after radiofrequency ablation. J Clin Gastroenterol 52(3):18-26

\section{Publisher's Note}

Springer Nature remains neutral with regard to jurisdictional claims in published maps and institutional affiliations.

\section{Submit your manuscript to a SpringerOpen ${ }^{\circ}$ journal and benefit from:}

- Convenient online submission

- Rigorous peer review

- Open access: articles freely available online

- High visibility within the field

- Retaining the copyright to your article

Submit your next manuscript at $\boldsymbol{\nabla}$ springeropen.com 